\section{DELAYED DYNAMIC IN GENRE EMERGENCE}

Pablo Amster, Dpto. de Matemática, FCEN, Universidad de Buenos Aires and IMAS, CONICET, Ciudad Universitaria (1428), Buenos Aires, Argentina. E-mail:

<pamster@dm.uba.ar>.

Bruno Mesz, Universidad Nacional de Quilmes, Roque Saenz Peña 352, Bernal, Buenos Aires, Argentina. E-mail:

$<$ Bruno.mesz@gmail.com>.

Juan Pablo Pinasco, Dpto. de Matemática, FCEN, Universidad de Buenos Aires and IMAS, CONICET, Ciudad Universitaria (1428), Buenos Aires, Argentina. E-mail:

<jpinasco@dm.uba.ar>.

Pablo H. Rodríguez Zivic, Dpto. de Computación, FCEN, Universidad de Buenos Aires, Ciudad Universitaria (1428), Buenos Aires, Argentina. E-mail: <prodriguez@dc.uba.ar>.

Submitted: <leave for Editor to date>

\begin{abstract}
In this work we present a model of style emergence based on consensus models. Given a set of agents, each one with its own style modeled by some real number between 0 and 10 , we define a network of relationships; two agents are connected when the distance of their styles is lower than certain fixed value. Only connected agents can interact, although the influence is not based on the present values of their styles but on the values they had some time ago, that is, their history or tradition. The system reaches several non-interacting genres or style clusters, and this dynamic yields more styles than the ones obtained without the influence delay. (Keywords: flocking, evolution, networks, musical styles).
\end{abstract}

\section{Introduction}

In the second half of the $19^{\text {th }}$ century several new popular music genres emerged across the Americas, like Blues in the south of United States, Candombe in Uruguay and Argentina, Habanera in Cuba, Samba in Brazil, or Tango in Argentina, to mention only a few. All of them display a mixture of characteristics from European and African music. As E. Sábato said [13],

It may be painful that history is always novel and therefore invariably confusing and inclined to mixtures. But that is what makes it so exciting. The self-identity is to be found in Logic or Mathematics: nobody can request History to produce something as pure (and also boring) as a cone or a sine wave. The inevitable part of the hybridity is always fruitful: enough to think of the Gothic style, and the black music in the United States.

Several works analyzed the influence of different musical traditions when they interact, and the apparition of new genres, see for example the article of Vega [19] about the origins of Tango, the one of Schuller [14] on the origins of jazz, the one of Tully [18] about Blues, or the work of Szwed and Marks [16] analyzing the transformation of the European dances due to Afro-american influences.

In this work, we simulate the evolution of genres in a system of interacting agents. Each agent represents a different style, symbolized by a real number in the interval $[0,10]$. For example, a style may correspond in our model to a single musical feature such as the relative frequency of patterns of three con- secutive notes determining two specific intervals among all such three notes patterns in the music, which has been used to distinguish Baroque from Classical and Romantic styles by Rodriguez Zivic et al. in [12].

In this way, we are considering a single characteristic of musical genres. Of course, this is an oversimplification, although high-dimensional models can be considered with similar results.

We assume that the styles come from different origins, assigning the initial values at random. Note that each agent-style may correspond to the practice of a group of musicians and, conversely, a single musician may cultivate distinct styles. During some transient time, the agents modify slightly their values, which can be understood as a consequence of lack of instruments, individual explorations, new influences -or weakening of their old influences-.

Moreover, we construct a network structure among the agents, and we shall say that two agents are connected whenever the difference between their styles is less than some threshold value. This restriction accounts for the fact that only those features of African and European music that were significantly similar took part in the hybridization process that gave origin to genres such as jazz or tango. For instance, in the case of jazz, the more complex polyrhythmic patterns of African music did not survive, and only the simplest polyrhythms interacted with European regular rhythmic schemas to generate the syncopated style of early jazz [14]. In the multidimensional case, musical measures of similarity, combining various rhythmic, melodic, harmonic and timbre parameters may be used to determine the threshold of connectedness, see [1].

Observe that this restriction arose also as a consequence of the local character of interactions, and the fact that, in most cases, only musicians playing in the same geographical area could interact and transfer their knowledge and traditions, see for example [8]. Today, mass media, internet and different communication tools enable the musicians to connect across large geographical distances, and several works where the networks of musicians and genres were modeled by using on-line stores, collaborations among musicians, and preferences of people, see [4], [7], [11]. However, the interplay of musicians in America during the $19^{\text {th }}$ century was strongly dependent on their physical interaction, due to the lack of sheet music and recording devices among non-reading musicians from popular sectors, see [3], [15].

Finally, a crucial aspect in the interactions in this model is the existence of temporal delays. Delays reflect the fact that, when musicians interact, they interchange some characteristics not just of their present style but also of their past styles and their musical traditions.

The evolution of styles is similar to opinion dynamic models with a continuum of opinions [6], [17], and flocking models [5], [20]. Each agent replaces its own style with a weighted average of the styles of their neighbors. The system evolves until some equilibrium is reached, with all the agents concentrated on few genres. However, the number of different genres in the final state is greater than the number of genres with the same initial conditions and a non-delayed dynamics. 
We wish to stress that delayed interactions were studied in flocking and swarming models (see for example [2], [10]), since the coordination of robots for exploration must consider the communication delay. However, in flocking models we have an additional repulsive interaction, since the birds, fishes or robots try to avoid collisions. So, the effect observed here is new, and it could be of interest in other models.

On the other hand, the introduction of the temporal delay makes no sense in opinion dynamic models. In fact, nobody will try to reach a consensus over an opinion that he had had in the past, only the current opinions are of interest. We wish to mention the beautiful paper Motsch and Tadmor [9], where the effect of heterophilia was introduced in opinion models, namely, an agent gives more weight to opinions that are far away from its own opinion inside the region of influence. In this case, the system stabilizes with fewer clusters than in the constant weight case, which is the opposite phenomenon than the one described here.

\section{The model}

We consider here a discrete model. We start with $N=\{1, \ldots, n\}$ agents, each one of them with initial value $x_{\mathrm{i}}(0)$, a real number in some interval [a,b] for $i=1: N$. We fix an integer $d$, and allow the agents to vary their value at random during $d$ steps. Hence, each one has a random trajectory $x_{\mathrm{i}}(k)$ for $k=1: d$ (with small variance, not allowing the agents to travel more a distance $r$ in mean). Moreover, each agent has a randomly assigned delay $d_{\mathrm{i}}<d$ which can be allowed to vary with time at random.

Given the mean distance $r>0$ of the initial excursions, we have an evolving network of connected agents, with connectivity matrix $A(k)$, where

$$
A_{\mathrm{ij}}(k)=A_{\mathrm{ji}}(k)=1 \quad \text { if }\left|x_{\mathrm{i}}(k)-x_{\mathrm{j}}(k)\right|<r,
$$

and zero otherwise. The degree $c_{\mathrm{i}}$ of the node corresponding to agent $i$ is equal to the number of agents satisfying that their values are at distance less than $r$. This matrix will change at each step of the dynamic. We assume that the number of agents is large enough in order to have a connected network. A different choice, usual in the flocking literature, consists in taking a fixed number $m$ of neighbors, the closest ones (in this case it must be decided if long-range interactions are allowed, or a minimum distance $r$ is also imposed).

We use the variable $k$ as before to index the temporal steps, and for each step $k>d$, we update the values of each agent according to the following rule:

$$
x_{\mathrm{i}}(k+1)=x_{\mathrm{i}}(k)+\sum_{j \neq i} c_{\mathrm{ij}} g\left(x_{j}, x_{i}, d_{j}, d_{i}\right) f\left(\left|x_{\mathrm{i}}(k)-x_{\mathrm{j}}(k)\right|\right)
$$

where $f(s)=1$ if $|s|<r$, and zero otherwise, and $g$ defines the dynamic. Several alternative dynamics are possible: the delay can be applied to agent $i$ (who compares the actual value of agent $j$ with his own value at time $k-d_{\mathrm{i}}$ ), or to agent $j$, (who shares its value $x_{\mathrm{j}}\left(k-d_{\mathrm{j}}\right)$ with agent $\left.i\right)$. It can be assigned at random in each step, or it can depend on each couple of agents $i$ and $j$. In the case of non-delayed dynamics we take $d_{\mathrm{i}}=0$ for $i=1: N$.

The parameter $c_{\mathrm{ij}}$ is the intensity of the coupling between agents $i$ and $j$. Here, we choose $c_{\mathrm{ij}}=N^{-1}$ as in [20], however, the present one is a non-linear system, and we cannot apply directly their results. Another frequent choice is $c_{\mathrm{ij}}=c_{\mathrm{i}}^{-1}$, the inverse of the degree of the node corresponding to agent $i$. The system evolves until some stationary state is reached. We recall that, for non-delayed dynamics, this result was proved in [6], and that the number of final genres cannot be predicted even in this case. Moreover, the trajectories $x_{\mathrm{i}}(k)$ can display an oscillatory behavior in the presence of temporal delays, at least for some particular cases; so we must rely on the numerical simulations.

In the next section we discuss the parameters and the results of the numerical simulations.

\section{Simulations}

We performed 5000 simulations of each model, with $N=90$ agents, a random uniform distribution of the initial values $x_{\mathrm{i}}(0)$ in $[0,10], d=80$ steps, and distance $r=1$. After a random update of the agent's values in those steps, an individual delay $d_{\mathrm{i}}<d$ was assigned to each agent.

It remains to choose a function $g$ governing the dynamical system. In our simulations we used four different functions $g$,

$$
\begin{array}{lll}
\text { 1. } & \text { ND: (no delay) } & g=x_{\mathrm{j}}(k)-x_{\mathrm{i}}(k) \\
\text { 2. } & \text { RD: (random delay) } & g=x_{\mathrm{j}}\left(k-d_{j}(k)\right)-x_{\mathrm{i}}(k) \\
\text { 3. } & \text { Dj: (agent's j delay) } & g=x_{\mathrm{j}}\left(k-d_{j}\right)-x_{\mathrm{i}}(k) \\
\text { 4. } & \text { Di: (agent's i delay) } & g=x_{\mathrm{j}}\left(k-d_{i}\right)-x_{\mathrm{i}}(k)
\end{array}
$$

In model 2.RD, the delay $d_{\mathrm{j}}(k)$ was randomly changed at each step for each player, in contrast to models 3.Dj and 4.Di where the delay was randomly initialized and held constant through the iterations.

The behavior of the four models was different, in Figure 1 we show the histogram with 5000 realizations of each model. Clearly the number of styles in delayed models when the simulations stop is greater than in the case of no delay. Moreover, the mean, median and mode of each model are given in Table 1 , and show less genres for delayed dynamic than in the other cases.

Starting all of the models with the same initial condition in the

Figure 1. Histogram of the number of final genres.

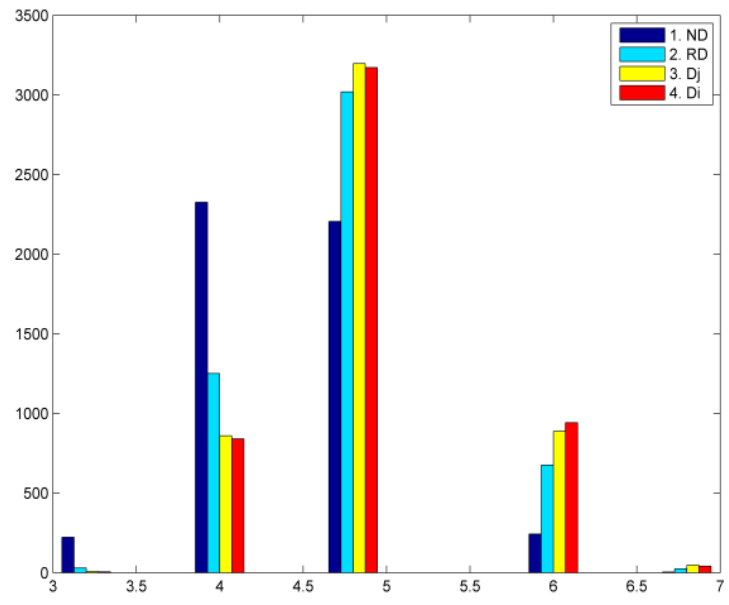


Fig. 2._Boxplot of the number of genres per model type.

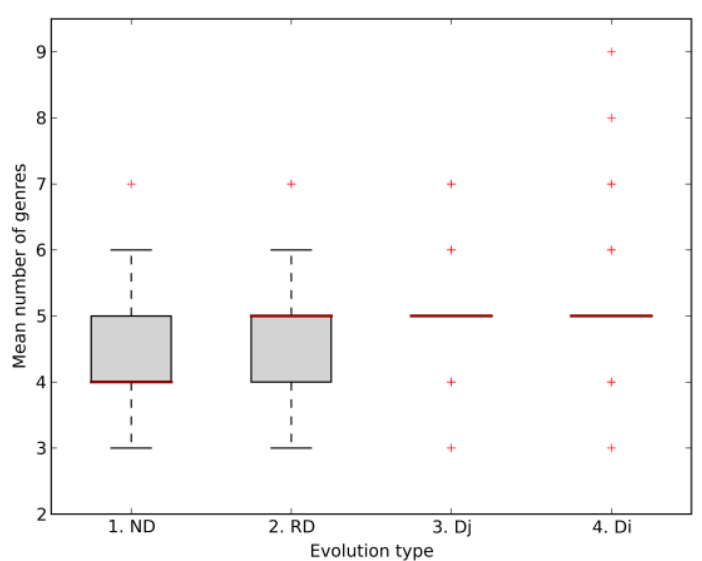

Table 1. Means, medians and modes for each of the models

\begin{tabular}{|l|l|l|l|l|}
\hline & 1.ND & 2.RD & 3.Dj & 4.Di \\
\hline Mean & 4.49 & 5.02 & 4.88 & 5.03 \\
\hline Median & 4 & 5 & 5 & 5 \\
\hline Mode & 4 & 5 & 5 & 5 \\
\hline
\end{tabular}

first 80 steps, in a single realization each model can generate more styles than the other ones. However, the performance of the non-delayed model is clearly different, giving more genres only in a small percentage of cases.

We compared statically the yielded number of genres of each run. Specifically, we compare means using a t-test, and medians using the Wilcoxon rank-sum test. Results show the mean and median number of genres yielded by the 1.ND model is less than the delayed versions. Also, the 2.RD model yields significant less genres than the 3.Di and 4.Dj. Lastly, there is a marginally significant difference between 3.Dj and 4.Di. See the boxplot in Figure 2.
Fig. 3._Evolution of styles. Left panel: delayed dyna mic. Right panel: non delayed dynamic.
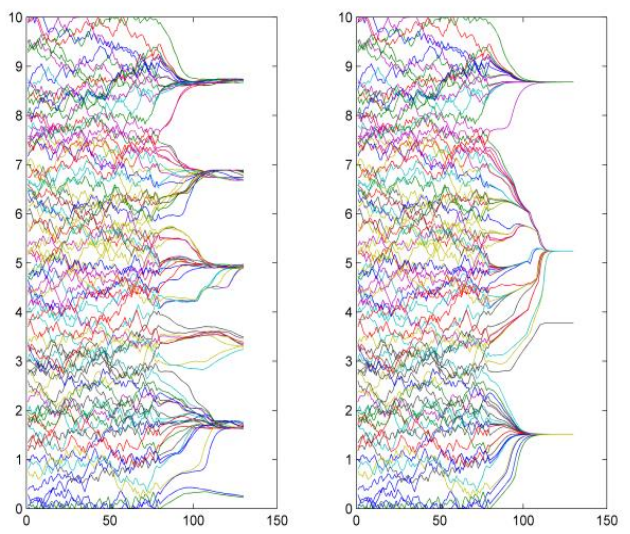

Comparison between the delayed models 2.RD and 3.Dj shows that both give the same number of styles on 3354 simulations (67.08\%), 2.RD gives more styles in $1156(23.12 \%)$, and 3.Dj gives more styles in 490 (9.8\%).

In Figure 3 we show the trajectories of each agent, in the right hand side we observe a realization of the evolution of the system with the non-delayed dynamic; in the left hand side we have a realization of model 4.D.

Let us consider now the evolution of the network of agents. The dynamic produces local consensus, disconnecting the network in several groups, and each one tends to be fully connected. We show a few stages of this process in Figure 4 (the values in axis $y$ are randomly chosen to split the agents).

Observe that, when a given group of agents $\left\{x_{\mathrm{i}}\right\} i \varepsilon I$ satisfy that all the distances between them are lower than $r$, and no one interacts with the remaining agents $N \backslash I$ in some temporal interval $[T, T+d]$, they will remain isolated forever.

\section{Fig. 3. Snapshots of the network evolution.}
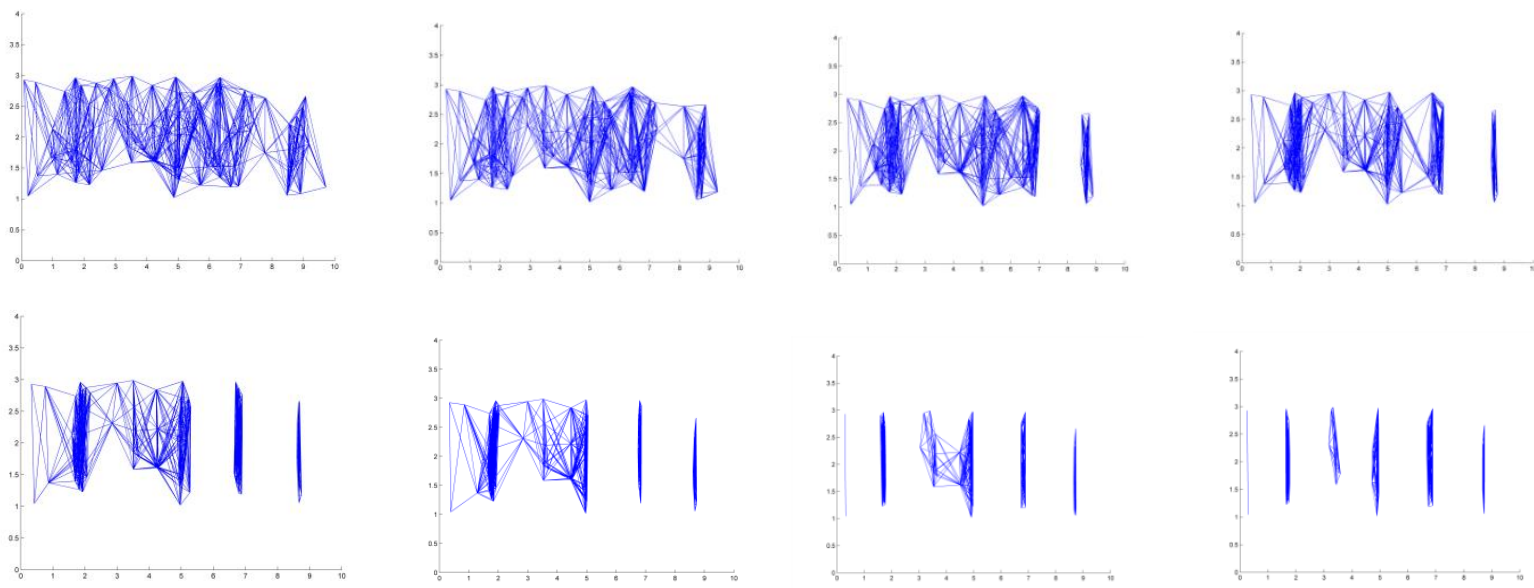
Their values will change due to the reciprocal influences, and they will remain bounded between the values

$$
\begin{aligned}
& m=\min \left\{x_{\mathrm{i}}(k): i \varepsilon I, k \varepsilon[T, T+d]\right\}, \\
& M=\max \left\{x_{\mathrm{i}}(k): i \varepsilon I, k \varepsilon[T, T+d]\right\} .
\end{aligned}
$$

We can observe that, for one of those agents, the update of its value is given by

$$
\begin{aligned}
x_{\mathrm{i}}(k+1) & =x_{\mathrm{i}}(k)+N^{1} \sum_{j \varepsilon I, j \neq i}\left[x_{\mathrm{j}}\left(k-d_{j}\right)-x_{\mathrm{i}}(k)\right] \\
& =x_{\mathrm{i}}(k)+N^{1} \sum_{j \varepsilon I, j \neq i} x_{\mathrm{j}}\left(k-d_{j}\right)-N^{1} \sum_{j \varepsilon I, j \neq I} x_{\mathrm{i}}(k) \\
& =N^{1}\left[(N-|I|+1) x_{\mathrm{i}}(k)+\sum_{j \varepsilon I, j \neq I} x_{\mathrm{j}}\left(k-d_{j}\right)\right],
\end{aligned}
$$

and then we have

$x_{\mathrm{i}}(k+1) \geq N^{1}\left[(N-|I|+1) m+\sum_{i \varepsilon I, j \neq I} m\right]=m$

$x_{\mathrm{i}}(k+1) \leq N^{-1}\left[(N-|I|+1) M+\sum_{i \varepsilon I, j \neq I} M\right]=M$.

We are not specifying the exact delayed dynamic since the result is valid for all of them whenever the delays belong to $[0, d]$.

A technical argument enable us to show that in the following $d$ temporal steps, the value $x_{\mathrm{i}}$ will be strictly greater than $m$ (resp., less than $M$ ), unless all the values involved in the computation are equal to $m$ (resp., $M$ ). In particular, we deduce that the values of agent $i$ must be all equal to $m(M)$ in $[T+d, T+2 d]$, and the convergence is proved by using an induction argument similar to the one in [2].

So, whenever a complete graph (i.e, a fully connected component of the network) appears, it will remain completely connected forever and the values of those agents tend to be closer. In Figure 3 we show some steps of the network evolution.

\section{Conclusions}

We have presented a simple model of musical genres evolution with temporal delays, based on continuous opinion models. Each agent represents a style, and we may consider that closer styles will contain some common musicians, influencing (and influenced by) other musicians in those styles.

In the process of the emergence of African-American musical genres, African musicians were forcibly removed from their native geographic and cultural environments; in this context, it is reasonable to assume that they experienced a certain stylistic drift due to isolation from their cultures of origin, along with the observable fact [14] that interactions in their new locations were based on the elements of their musical traditions (rhythm, melodic and harmonic features, instruments, techniques), which were transferred or mixed with those of other musicians. Later, the temporal delay appears as an individual search on their own history.

We have shown the existence of several local consensus, well defined or mature styles, appearing as a mix of different initial styles. This result is obtained also with non-delayed dynamics, although in this case a lower number of genres are obtained.

\section{References and Notes}

* This paper was presented as a contributed talk at Arts, Humanities, and Complex Networks $-4^{\text {th }}$ Leonardo satellite symposium at NetSci2013. See <http://artshumanities.netsci2013.net>.

1. J-J. Aucouturier, F. Pachet." Representing Musical Genre: A State of the Art”, Journal of New Music Research, Vol. 32, No. 1, (2003), pp. 83-93

2. V. D. Blondel, J. M. Hendrickx, A. Olshevsky, J. N. Tsitsiklis, "Convergence in multiagent coordination, consensus, and flocking," Decision and Control 2005, pp. 2996-3000; and 44 ${ }^{\text {th }}$ IEEE European Control Conference, 2005.

3. P. V. Bohlman, "Immigrant, folk, and regional music in the twentieth century", in D. Nicholls, ed. The Cambridge History of American Music, (Cambridge, U.K.: Cambridge Univ. Press, 1999).

4. N. J. Bryan, G. Wang, "Musical influence network analysis and rank of sample-based music," 12th International Society for Music Information Retrieval Conference (ISMIR 2011)

5. F. Cucker, S. Smale, "The mathematics of emergence," Japanese Journal of Mathematics 2, (2007) pp. 197-216.

6. R. Hegselmann, U. Krause, "Opinion dynamics and bounded confidence models, analysis and simulation," Journal of Artificial Societies and Social Simulation 5, No. 3 (2002) < http://jasss.soc.surrey.ac.uk/5/3/2.html $>$

7. R. Lambiotte, M. Ausloos, " Uncovering collective listening habits and music genres in bipartite networks," Phys. Rev. E 72 (2005) pp. 066107-066118.

8. J. C. Lena, R. A. Peterson, "Classification as Culture: Types and Trajectories of Music Genres," American Sociological Review 73 No. 5 (October 2008) pp. 697-718.

9. S. Motsch, E. Tadmor, "Heterophilious dynamics enhances consensus", preprint 2013, <http://arxiv.org/abs/1301.4123>

10. U. Munz, A. Papachristodoulou, F. Allgower, "Delay-dependent rendezvous and flocking of large scale multi-agent systems with communication delays," Decision and Control 2008, pp. 2996-3000; and $47^{\text {th }}$ IEEE European Control Conference, 2007.

11. J. Park, O. Celma, M. Koppenberger, P. Cano, J. M. Buldú, "The Social Network of Contemporary Popular Musicians," Int. J. Bifurcation and Chaos 17 No. 7 (2007) pp. 2281-2288

12. P. H. Rodriguez Zivic, F. Shifres, G. A. Cecchi. "Perceptual basis of evolving Western musical styles", To appear in Proceedings National Academy of Sciences, www.pnas.org/cgi/doi/10.1073/pnas.1222336110 (2013)

13. E. Sabato, "Hibridaje", in Tango, discusión y clave (Buenos Aires, Ed. Losada, 1963).

14. G. Schuller, Early Jazz: Its Roots and Musical Development. Oxford University Press. 1968.

15. E. Southern, The Music of Black Americans, W. W. Norton \& Company, Inc.

16. J. F. Szwed, M. Marks, "The Afro-American Transformation of European Set Dances and Dance Suites," Dance Research Journal 20 No. 1 (1988) pp. 29-36.

17. G. Toscani, "Kinetic models of opinion formation," Communication in Mathematical Sciencies 4, No. 3 (2006) pp. 481-496.

18. S. J. Tully, "The Triumph of the Blues Form," Perspectives in History 18 (2002/2003) pp. 37-44.

19. C. Vega, "Las especies homónimas y afines de 'Los orígenes del tango argentino’," Revista Musical Chilena 21, No. 101 (1967) pp. 49-65.

20. T. Vicsek, A. Czirok, E. Ben-Jacob, I. Cohen, O. Schochet. "Novel type of phase transitions in a system of self-driven particles," Physical Review Letters 75, No. 6 (1995) pp. 1126-1229. 